\title{
Comparison of Drug Related Problems Associated with Use of Narrow Therapeutic Index Drugs and Other Drugs in Hospitalized Patients
}

\author{
Kapil lyer ${ }^{1 *}$, Neha Dilipkumar ${ }^{1}$, Sharmin Vasaya', Sunita Pawar ${ }^{1}$, Arundhati Diwan ${ }^{2}$ \\ 'Department of Clinical Pharmacy, Poona College of Pharmacy, Bharati Vidyapeeth (Deemed to be) University, Pune, Maharashtra, INDIA. \\ ${ }^{2}$ Department of General Medicine, Bharati Hospital, Pune, Maharashtra, INDIA.
}

\begin{abstract}
Objectives: To identify and categorize types of Drug Related Problems (DRPs), assess various risk factors for developing DRPs and delineate if drugs with narrow therapeutic index or non-narrow therapeutic index drugs cause greater number of DRPs. Methods: Patients from General Medicine, Surgery, Psychiatry, Cardiac and Intensive Care Unit (ICU) departments of a tertiary care teaching hospital were included. Data was collected prospectively over eight months from medical history, medication charts, case notes and laboratory data. Only those patients prescribed at least five drugs were included. Pediatric and Oncology patients and pregnant women were excluded from the study. Narrow therapeutic index (NTI) drugs (Insulin, digoxin, warfarin, levothyroxine, aminoglycoside antibiotics, carbamazepine, lithium and phenytoin) were compared with non NTI-drugs to delineate patterns of DRPs between both groups. Results: A total of 200 patients were enrolled in the study, and 172 DRPs were identified. The most common DRPs were: drug interactions $(63 \%)$, inappropriate drug use (10.5\%) and adverse drug reactions (10.5\%). Polypharmacy and duration of hospitalization were established as significant risk factors for
\end{abstract}

developing DRPs. NTI-drugs had a greater risk of developing DRPs (0.22) versus non-NTI drugs (0.08). Two categories of DRPs were found to be more significantly associated with NTI-drugs: ADRs and inappropriate drug use. Conclusion: While ADR and inappropriate drug use were more common with NTI-drugs, in clinical practice other non NTI-drugs: Anti-psychotic drugs (quetiapine, amisulpride etc.) and NSAIDs (aspirin) showed a high tendency for interactions and needed frequent monitoring.

Key words: Drug related problems, Drug risk ratio, Narrow therapeutic index drugs, Polypharmacy

Correspondence

Kapil lyer, PharmD Intern, Department of Clinical Pharmacy, Bharati Hospital, Pune-411046, Maharashtra, INDIA.

Phone: +91-9763239960

Email: kapil.iyer999@gmail.com

DOI: 10.5530/jyp.2018.10.70

\section{INTRODUCTION}

Drugs are dualistic therapeutic tools which come with distinctive benefits but diverse risks. ${ }^{1}$ The intended benefits of therapy may sometimes be eclipsed by unintended problems. Drug Related Problems (DRPs) are any such event or circumstance involving drug therapy, which may actually or potentially interfere with desired health outcome. ${ }^{2}$ If overlooked, DRPs are serious complications representing significant morbidity, mortality and economic liability. Multiple worldwide studies show high evidence of drug related problems occurring in hospitalized patients..$^{3-4}$ However, it has been also been corroborated that upto $50 \%$ of these are potentially preventable. ${ }^{5}$

Generally, female sex, polypharmacy, renal impairment, age over 65 years and use of narrow therapeutic index (NTI) drugs were identified as risk factors for DRPs. NTI drugs call for greater attention, because although identified as cause for nearly one-third of emergency department visits, ${ }^{6}$ very few studies have established the exact nature of DRPs with use of NTI drugs.

In India, DRPs have been researched to derive their impact on society, but no studies specifically characterize NTI drugs as a cause. In this study, we have attempted to identify and categorize the types of DRPs among hospital in-patients, identify their risk factors and focus on problem areas that require greater caution with NTI drugs as compared to non NTI drugs.

\section{METHODS AND MATERIALS}

\section{Study Design}

The prospective, observational, cohort, comparative study was designed to investigate and compare the patterns of drug related problems occurring in the in-patients of a tertiary care teaching hospital, between patients receiving narrow therapeutic index drugs and those that do not. The institutional ethics committee of Bharati Medical College and Hospital approved the protocol to conduct the study. Informed consent was obtained from all patients. Patients hospitalized between September 2016 and March 2017 (six months) were enrolled. A total of 200 patients (equal number of patients receiving NTI and non-NTI drugs) were enrolled from General Medicine, Surgery, Orthopedics, Cardiology and Psychiatry. Patients between the age of 18-90 years and of either gender with upto three co-morbid conditions such as cardiovascular diseases, diabetes mellitus, renal or hepatic impairment were included. Patients prescribed with drugs having a narrow therapeutic index and/or patients prescribed with non narrow therapeutic index drugs were included. Based on literature survey and an initial pilot study, we included the following NTI-drugs: insulin, digoxin, warfarin, levothyroxine, aminoglycosides, carbamazepine, lithium and phenytoin in this study. The following patients were excluded: Patients prescribed with less than five drugs, patients admitted to emergency medicine ward, gynaecology and pediatric departments, patients undergoing chemotherapy, patients 
treated in out-patient departments and patients that have been re-admitted for same complaints.

The following data were recorded for each patient: demographic details, presenting complaints, medication charts, medical/medication history, case notes and laboratory tests. Data was collected from various sources including the patient files, interviews with patient and family members (with permission), other healthcare professionals (nurse, physician etc.) and laboratory investigation reports and recorded on a self-designed data collection form.

\section{Data interpretation}

Individual patient cases were analyzed and DRPs were identified. Pharmaceutical Care Network Europe (PCNE) classification system of DRPs (v7.0) was used to assess and identify DRPs with prior approval. However, the PCNE tool classified the DRPs into 36 possible categories for causes. Hence for ease of calculation, DRPs identified were categorized into eight main categories: Adverse drug reactions (ADRs), Drug Interaction (DI), Subtherapeutic dose, High Dose, Inappropriate drug use (wrong route/frequency of administration, wrong time of administration), Unnecessary drug treatment (drug not required clinically), Untreated indication, Patient related factors. Potential ADRs were assessed using standardized scales such as Naranjo's Scale for Causality, Hartwig-Siegel Scale for assessment of Severity of the ADR and Modified Schumock-Thornton Scale for Preventability.

The standard database Micromedex was used to cross-verify prescribed doses, treatment regimen and to check for drug-drug, drug-food, druglaboratory interactions. All identified DRPs were then documented in the PCNE DRP registration form and any discrepancies promptly relayed to the resident/physician. In some cases, suggestions were recommended for optimizing drug therapy.

To identify which category of drugs are at higher risk to cause DRPs, the concept of drug risk ratio was calculated using the total number of DRPs caused by NTI/non NTI drugs divided by the total number of NTI/non NTI drugs prescribed to patients in the study.

\section{Statistical Analysis}

Microsoft Excel versions 2007/2010 were used to perform database entries for collected data. Descriptive statistics were described as means and frequencies with standard deviations or standard errors using MS Excel. Data was accepted as statistically significant if P-values was less than $0.05(p<0.05)$. To compare differences in demographics between NTI and non NTI users groups, unpaired T tests were used, while chi-squared tests were used to assess other risk factors.

\section{RESULTS}

The study included 200 in-patients in which 67\% male and 33\% female patients were included between the age groups of $18-95$ years (mean age: 47.75 years, SD: 17.7 ). At the time of enrollment, $56 \%$ patients did not have any concomitant illnesses. $25.5 \%$ patients $(25.5 \%)$ presented with one co-morbidity, $13 \%$ with two (5.5\%) with three co-morbidities. Diabetes and Hypertension were found to be the most common co-morbidity.

The number of medications per patient ranged from 5 to 22 (mean 9.8 drugs with SD 3.9). 60\% patients were prescribed between 5-10 drugs, $26 \%$ between the range of $11-15$ drugs and $14 \%$ above 15 drugs. The average length of stay for the overall study population in terms of number of hospitalization days was found to be 6.7 days (with a SD 4.3), with approximately 1.8 (SD 1.45) DRPs identified per patient. The Demographic details of the NTI and Non-NTI drug users are mentioned in Table 1. A total of 172 DRPs were identified and classified into 8 broad categories based on the findings. The incidence of various categories of DRPs is given in Figure 1.

A total of 108 drug interactions were identified and 5 NTI drugs were found to have maximum potential for drug interactions: Digoxin, Amikacin, Levothyroxine, Theophylline and Phenytoin. The most commonly occurring drug-drug interactions are mentioned in Figure 2.

A total of 18 ADRs were identified of which $33 \%$ cases were preventable. The most common drugs observed as the cause of hospitalization were found to be insulin (16\%), clopidogrel (16\%), lithium (5\%) and olmesartan (5\%). The influence of various risk factors on the overall prevalence of DRPs (Table 2) was determined.

The 100 NTI-users used a total of 112 NTI-drugs, which represented $6 \%$ of the total number of drugs prescribed to 200 patients. Also, 100 nonNTI-drug users were included who used 1864 non-NTI-drugs (94\% of total drugs used). NTI-drugs with a risk ratio of 0.22 were found to be at greater risk to cause DRPs than non-NTI-drugs (drug risk ratio 0.08) $(\mathrm{p}<0.01)$ (Table 3$)$.

Table 1: Demographic details of NTI and Non-NTI users.

\begin{tabular}{cccc}
\hline & $\begin{array}{c}\text { NTI Users } \\
(\mathrm{N}=100)\end{array}$ & $\begin{array}{c}\text { Non NTI users } \\
(\mathrm{N}=100)\end{array}$ & Significance \\
\cline { 2 - 4 } & Mean (SD) & Mean (SD) & \\
\hline Age & $50.8(17.8)$ & $44.5(16.9)$ & 0.011 \\
No. of Drugs & $11.23(3.9)$ & $7.9(2.7)$ & $<0.001$ \\
No. of Days & $6.6(4.3)$ & $5.5(3.3)$ & 0.043 \\
No. of DRPs per patient & $0.25(0.34)$ & $1.47(1.5)$ & $<0.001$ \\
& $\%($ No.) & $\%($ No.) & \\
Females & $33 \%(33)$ & $33 \%(33)$ & 1 \\
Co - morbidities & & & \\
HTN & $35 \%(35)$ & $17 \%(17)$ & 0.006 \\
DM & $34 \%(34)$ & $9 \%(09)$ & $<0.001$ \\
\hline
\end{tabular}
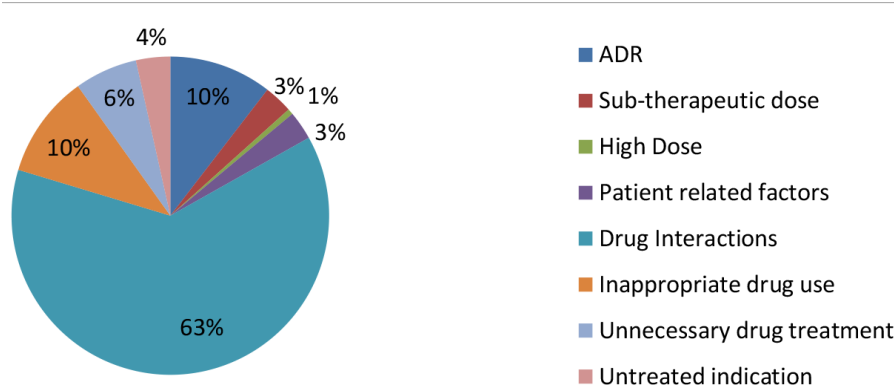

Figure 1: Incidence of DRPs identified in Hospitalized patients.

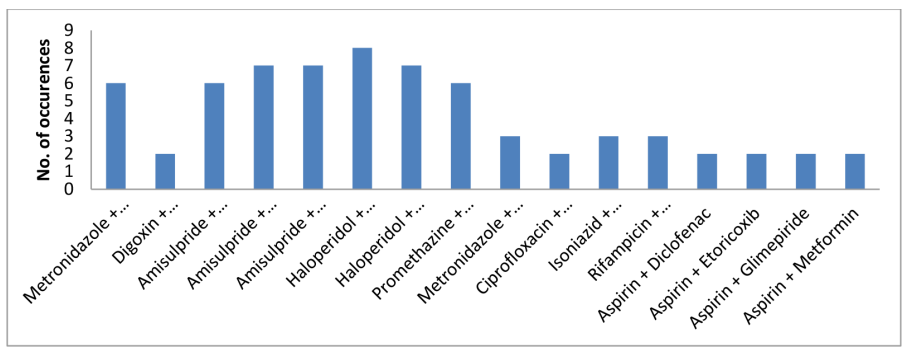

Figure 2: Frequency of major recurring Drug Interactions. 
Table 2: Influence of various risk factors on the overall prevalence of DRPs.

\begin{tabular}{|c|c|c|c|}
\hline $\begin{array}{l}\text { Risk parameters } \\
\text { for DRP }\end{array}$ & $\begin{array}{l}\text { Patients with } \\
\text { DRP } n(\%)\end{array}$ & $\begin{array}{l}\text { Patients without } \\
\text { DRP } n(\%)\end{array}$ & Significance \\
\hline \multicolumn{4}{|c|}{ Poly-pharmacy } \\
\hline$<10$ drugs $(\mathrm{N}=120)$ & $47(39.1)$ & $73(60.9)$ & 0.006 \\
\hline$>10$ drugs $(\mathrm{N}=80)$ & $48(60)$ & $32(40)$ & Significant \\
\hline \multicolumn{4}{|c|}{ Age } \\
\hline$<50(\mathrm{~N}=113)$ & $51(45.1)$ & $62(54.9)$ & 0.76 \\
\hline$>50(\mathrm{~N}=87)$ & $42(48.3)$ & $45(51.7)$ & Not significant \\
\hline \multicolumn{4}{|c|}{ Gender } \\
\hline Male $(\mathrm{N}=134)$ & $59(44)$ & $75(56)$ & 0.51 \\
\hline Female $(\mathrm{N}=66)$ & $33(50)$ & $33(50)$ & Not significant \\
\hline \multicolumn{4}{|c|}{ Length of Stay } \\
\hline$<7$ days $(\mathrm{N}=109)$ & $41(37.6)$ & $68(62.3)$ & 0.009 \\
\hline$>7$ days $(\mathrm{N}=91)$ & $51(56)$ & $40(44)$ & Significant \\
\hline \multicolumn{4}{|c|}{ Co-morbidities } \\
\hline One $(\mathrm{N}=52)$ & $25(48.07)$ & 27 (51.9) & 0.35 \\
\hline$>1(\mathrm{~N}=38)$ & $22(57.8)$ & $16(42.2)$ & Not significant \\
\hline
\end{tabular}

Table 3: Calculation of drug risk ratio.

\begin{tabular}{ccccc}
\hline & $\begin{array}{c}\text { Total no. of } \\
\text { drugs used }\end{array}$ & $\begin{array}{c}\text { No. of drugs } \\
\text { with DRPs (\%) }\end{array}$ & $\begin{array}{c}\text { Total no. } \\
\text { of DRPs }\end{array}$ & $\begin{array}{c}\text { Drug risk } \\
\text { ratio }\end{array}$ \\
\hline Non NTI drugs & 1864 & $196(10.5)$ & 147 & 0.08 \\
NTI drugs & 112 & $19(16.9)$ & 25 & 0.22 \\
$\quad$ Total & 1976 & $215(10.8)$ & 172 & $(\mathrm{p}<0.001)$ \\
\hline
\end{tabular}

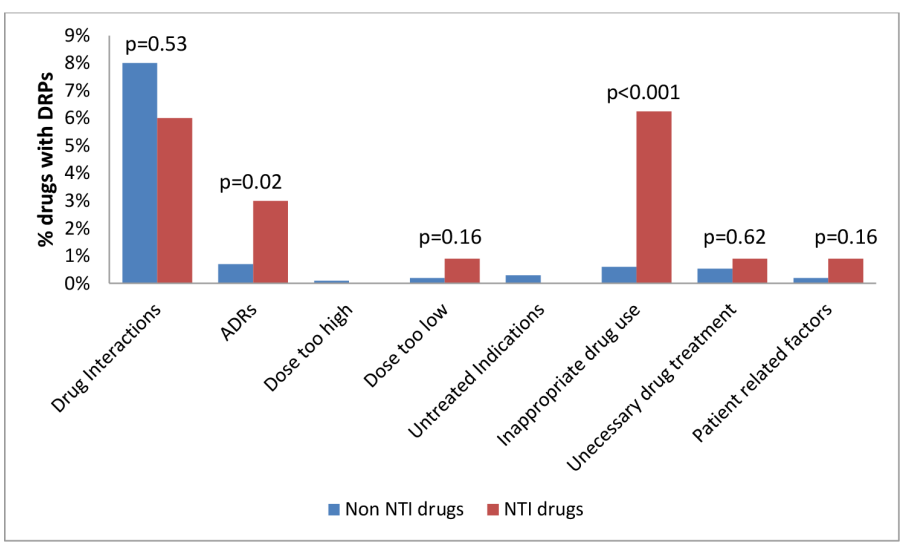

Figure 3: Comparison of the different types and frequencies of DRPs between NTI and non NTI drugs (NTI-drugs, N = 112 and non-NTI-drugs, $\mathrm{N}=1864$ ).

Incidence of ADRs and inappropriate drug use was found to be more significantly associated with NTI drugs (Figure 3).

\section{DISCUSSION}

This study was initiated to investigate various types of DRPs in hospitalized patients in a tertiary care teaching hospital in India. From data collected of 200 patients, a total of 172 DRPs were identified. These were then classified into 8 categories: drug interactions, inappropriate drug use (wrong time of administration, wrong route, wrong frequency), unnecessary drug treatment, untreated indication, ADR, dose too high, dose too low, patient factors (compliance, self-medication etc). The DRPs observed was lower than other similar studies. ${ }^{8-10}$ This could be because clinical pharmacists were actively involved in ward activities or an underestimate due to the lack of comprehensive history or knowledge about drugs taken etc. at the time of admission. Another study in India also noted similar discrepancies with results. ${ }^{11}$

The most commonly encountered DRPs in this study were drug-drug interactions (62.7\%). Several studies conducted in multiple countries showed that drug-drug interaction was the most majorly encountered type of DRPs. ${ }^{11-14}$ Most of the drug-drug interactions identified our study were only potential. However there were some cases that led to manifested adverse events such as hypokalemia, hypoglycemia, deranged LFTs and increased TSH. Although the study protocol did not mandate interventions, if any problem was identified, the physician was informed and asked to monitor the patients. In some cases, an alternative drug was suggested.

About $9 \%$ of the enrolled patients were found to have an ADR. This however, was found to be far lower than the results published by Harvard Medical Practice Study which showed 20\% of in-patients experiencing at least one ADR during their hospital stay. ${ }^{15}$ One-quarter of ADRs were due to NTI drugs and about $16 \%$ ADRs were due to insulin. ${ }^{6}$ Some of the identified ADRs were cephalosprin induced allergic reactions, hepatotoxicity due to anti-tuberculosis (TB) drugs, Statin induced arthralgias and anti-diabetic medications induced hypoglycemia.

In the attempt to evaluate the risk factors of DRPs, the results obtained by us corroborated published reports that the mean number of DRPs per patient was directly proportional to number of drugs used. In terms of polypharmacy, we found $60 \%$ patients receiving more than 10 drugs were found to have more DRPs. A number of studies concluded similar findings. ${ }^{12,14,16-17}$ However, DRPs were not found to have been significantly affected by gender and age. ${ }^{12,17}$

In our study, number of co-morbidities were not found to significantly affect the occurrence of DRPs in a patient, however, contrary to other similar studies, length of hospital stay in terms of days of hospitalization was found to be a significant contributory factor. ${ }^{18,29,20}$ Further research is warranted in this area to establish a stronger clinical link between DRPs and duration of hospitalization.

In our study, out of a total of 1976 drugs used, 112 were NTI drugs. On the whole NTI drug users were found to have higher mean age $(\sim 51$ years SD 17.8) compared to non NTI users (mean $\sim 45$ years \pm 16.9 ), were prescribed a higher average number of drugs per patients (11.23 as compared to 7.9 in the non NTI group), were hospitalized for more number of days as compared to the other drug users and had more number of DRPs. All of these parameters were found to be statistically significant. To assess the risk of NTI drugs as cause of DRPs, a drug risk ratio calculator was utilized. ${ }^{21}$ Based on this calculator tool, NTI drugs were found to have a higher association with occurrence of DRPs than non NTI drugs. Further investigating causes of DRPs in such groups, it was found that ADRs or inappropriate use of drugs commonly occurred with use of NTI drugs leading to DRPs.

These findings demonstrate that more attention is required for these specific problem areas when administering NTI drugs to patients. The nature of these problem areas (ADR and inappropriate use) is such that they are more likely to be associated with high risk medications and narrow therapeutic index drugs. In an effort to minimize the preventable DRPs, a more efficient DRP reporting system should be developed in health care settings. All healthcare professionals must be trained to identify the common risks for developing DRPs and design strategies to reduce these incidences. A clinical pharmacist could play an important role in reporting DRPs and performing clinical interventions to mitigate such cases. Various services such as patient counseling could also help 
reduce patient perception of drug therapy and reduce possibilities of developing DRPs due to practices such as self-medication. On the whole these findings do not indicate that only NTI drugs need be monitored. It was also observed that other drugs such as anti-psychotic drugs (amisulpride, quetiapine, haloperidol etc), NSAIDs (aspirin), ATT (rifampicin, pyrazinamide) and antibiotics (metronidazole and ciprofloxacin) were at higher risk to cause interactions and needed special monitoring in clinical practice.

A limitation in this study is that since the sample size (i.e. 200 patients) and study duration (8 months) was relatively short, the results might be an under evaluation of the real life statistics. Furthermore, since Therapeutic Drug Monitoring (TDM) was not performed in the hospital, these DRPs may be an under representation of DRPs.

\section{REFERENCES}

1. Reddena L, Sree Nagavalli K. Drug Therapy Problems: A review of Literature. Pharma Tutor. 2014;2(2):111-6.

2. Pcne.org [Internet]. Pharmaceutical Care Network Europe. The PCNE Classification V 7.0 [updated 5 Nov. 2017, cited July 2016]. Available from: http://www. pcne.org/working-groups/2/drug-related-problem-classification

3. Krahembuhl-Melcher A, Schilenger R, Lampert M, Haschke M, Drewe J, Krahembuhl S. Drug-related problems in hospitals: a review of recent literature. Drug Saf. 2007;30(5):379-407.

4. Bosma L, Jansman FA, Franken AM, Hartwig JW, Van den Bemt PA. Evaluation of Pharmacist clinical intervention in Dutch hospital setting. Pharm World Sci. 2007;30(1):31-8.

5. Kaufmann $C P$, Stämpfli D, Hersberger KE, Lampert ML. Determination of risk factors for drug-related problems: A multidisciplinary triangulation process. BMJ Open. 2015;5(3):e006376.

6. Budnitz DS, Shehab N, Kegler SR, Richards CL. Medication use leading to emergency department visits for adverse drug in older adults. Ann Intern Med. 2007:147(11):755-65.

7. Singh $\mathrm{H}$, Kumar BN, Dulhani N. The incidence and nature of drug-related hospital admission: A 6-month observational study in a tertiary health care hospital. J Pharmacol Pharmacother. 2011;2(1):17-20.

8. Algiriswami B, Ramesh M, Parthasarathi G, Basavanagowdappa H. A study of clinical pharmacist initiated changes in drug therapy in a teaching hospital. Indian J Pharm Pract. 2009;2(1):36-45.

9. Ganachari MS, Mahendra Kumar BJ, Shashikala CW, Fibin M. Assessment of drug therapy intervention by clinical pharmacist in tertiary care hospital. Indian J Pharm Pract. 2010;3(3):22-8.

10. Parthasarathi G, Ramesh M, Kumar JK, Madaki S. Assessment of drug related problems and clinical pharmacists' interventions in an Indian teaching hospital. J Pharm Pract Res. 2003;33(4):272-4.

11. Adepu R, Adusumilli PK. Assessment of Drug Related Problems in Patients with Chronic Diseases through Health Status Survey in a South Indian Rural Community Setting. Indian J Pharm Sci. 2016;78(4):537-42.

12. Yvonne K, Moideen-Kutty FB, Li SC. Drug-related problems in hospitalized patients on polypharmacy: the influence of age and gender. Ther Clin Risk Manag. 2005;1(1):39-48.

13. Zaman HH, Hoo FW. Drug-related problems in type 2 diabetes mellitus patients with hypertension: A cross sectional study. BMC Endocrine Disorders. 2013;13(1):1192.

14. Movva R, Jampani A, Nathani J, Pinnamaneni SH, Challa SR. A prospective study of incidence of medication-related problems in general medicine ward of a tertiary care hospital. J Adv Pharm Technol Res. 2015;6(4):190-4.

15. Brennan TA, Leape LL, Laird NM, Hebert L, Localio AR, Lawthers AG, et al. Incidence of adverse events and negligence in hospitalized patients. Results of the Harvard Medical Practice Study I. N Engl J Med. 1991;324(6):370-6.

16. Strand LM, Morley PC, Cipolle RJ, Ramsey R, Lamsam GD. Drug-related problems: Their structure and function. Ann Pharmacother. 1990;24:1093-7.

17. Blix HS, Viktil KK, Reikvam A, MogerTA, Hjemaas BJ, Pretsch P, et al. The majority of hospitalised patients have drug-related problems: Results from a prospective study in general hospitals. Eur J Clin Pharmacol. 2004;60(9):651-8.

18. Tigabu B, Daba D, Habte B. Drug-related problems among medical ward patients in Jimma University specialized hospital, Southwest Ethiopia. J Res Pharm Pract. 2014;3(1):1.

19. Ayalew MB, Megersa TN, Mengistu YT. Drug-related problems in medical wards of Tikur Anbessa specialized hospital, Ethiopia. J Res Pharm Pract. 2015;4(4):216-21.

20. George J, Munro K, McCaig D, Stewart D. Risk factors for medication misadventure among residents in sheltered housing complexes. $\mathrm{Br} \mathrm{J}$ Clin Pharmacol. 2007;63(2):171-6.

21. Blix HS, Vitkil KK, Moger TA, Revikam A. Drugs with narrow therapeutic index as indicators in the risk management of hospitalized patients. Pharm Pract (Granada). 2010;8(1):50-5.

Article History: Submission Date : 02-12-2017; Revised Date : 19-02-18; Acceptance Date : 02-04-18.

Cite this article: Iyer K, Dilipkumar N, Vasaya S, Pawar S, Diwan A. Comparison of Drug Related Problems Associated with Use of Narrow Therapeutic Index Drugs and Other Drugs in Hospitalized Patients. J Young Pharm. 2018;10(3):318-21. 